International Egyptian Journal of Nursing Sciences and Research (IEJNSR)

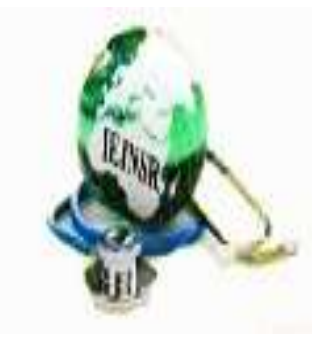

Review article

Received 2021-05-15

Accepted 2021-05-19

Published 1- 7-2021

\title{
The benefits of Kegel's exercises during pregnancy
}

\author{
Authors: Lawahez Mabrouk Dwedar \\ lecturer of Women's Health and midwifery Nursing, Faculty of Nursing Kafrelsheikh University, Kafrelsheikh, \\ Egypt
}

*Corresponding author: drlawahezmabrouk@gmail.com

\begin{abstract}
Antenatal Kegel's exercise as a type of conservative intervention is a safe and an effective technique that help the muscles control well during gestation. Kegel's exercise, as a type of conservative intervention, is a safe and effective technique that restores or develops pelvic floor muscle strength and can be practicing during gestation. This helps women control this musculature during pregnancy and during the expulsive phase of labor. It was defined as a program of repeated voluntary pelvic floor muscle contractions and should be corroborative by health professional for they may play an important role in beneficent the fineness of women's life in prenatal, perinatal and postnatal period. Women's health and maternity nurses play a vital role in health promotion, screening, diagnosis and prevention of complication for pregnant women. The nurse have role in paying more attention to Kegel's exercises for women in antenatal period in order to reduce complications related to labor and delivery outcome and thus enhancing quality of women's lives during antenatal, perinatal and the postpartum period
\end{abstract}

Keywords: Antenatal, Kegel's exercises, pregnancy

\section{INTRODUCTION}

Moderate and regular antenatal exercise during pregnancy period is likely to improve course of pregnancy and easier labor when compared with that, of a sedentary lifestyle. So, The American College of Obstetricians and Gynecologists, International Consultation on Incontinence (ICI), the UK's National Institute for Health and Clinical Excellence (NICE) guidelines which recommend that all women in pregnancy should be demonstrating exercise during antenatal period, if women 25 didn't have any complication during pregnancy period (1).

The pelvic floor muscles are involved in many functions as like of support the pelvic, abdominal organs, uterus, the bladder, and the bowels. In addition to their role in the sexual function (2). There are many factors such as pregnancy, vaginal delivery, childbirth, aging, and weight gain are weaken the pelvic floor muscles and decrease its tone, which leading to negatively impact on woman's health as physical, sexual and professional activities for 
example stress urinary incontinence and prolapse of genital tract organ (3).

\section{Definition of Kegel's exercises}

Alternative names for pelvic floor exercises (PFEs), pelvic muscle strengthening exercises. Kegel exercises were originally developed by Dr. (Arnold Kegel in 1948) as a method of controlling incontinence in women following childbirth and defined the Kegel's exercises as doing by contracting and relaxing pelvic floor muscles repeatedly $(4,5)$.

\section{The benefits of kegel's exercise}

Delivery by vaginal has been released as very important factor that it can destroy the pelvic floor muscles and so reduced or alter the supporting building in the pelvis floor muscles. Within this condition, pelvic floor muscle training may be an auxiliary tool for alleviating the musculoskeletal changes looked during pregnancy and the postpartum period, which is also connected with urinary incontinence. kegel's exercise is aimed at strengthening the indented musculature that is part of the striated urogenital sphincter muscle accountable for the stoppage of the urethral lumen after correct contraction of the pelvic floor muscles (6).

The benefits of kegal's exercise training for women during third trimester of pregnancy period are very significant benefit in woman life such as; help woman to maintaining bladder and bowel control, preventing and treating urinary incontinence in late pregnancy, also helping in strengthen muscles of vagina, uterus and bowel, protecting PFM from weakness and disorders by improve strength and function of the pelvic floor muscles, and helping in lubricate vagina during sexual arousal Also, it helps in healing after childbirth surgery in woman and increase quality of women's life quality (7).

The researchers founded that Kegel's exercise training is an essential part in rehabilitation programs of pelvic girdle pain postpartum. In addition the researchers added that, the scientific guide in considerate to the efficiency of pelvic floor muscle training program in treating leakage of urine and ameliorative the woman's quality of life (8).

A (2017) study, the results revealed that pelvic floor muscles exercise is a significant in removing slight, primary and various symptoms during the perimenopausal age; helping in lowering of the most symptoms such as anxiety and depression; progress confidence, intimacy, sexual satisfaction, and the strength to extent orgasm and helping in decreasing pelvic organ prolapse (9).

\section{Side effects of Kegel's exercises}

Kegel exercises may be having side effects when the person not done correctly, as run over or not contracting the proper muscles, it can lead to problems as urinary incontinence, a weaker the pelvic floor, pelvic organ prolapse, a urinary tract infection and back pain (10).

\section{Contraindication of doing kegel's exercise}

pelvic health solutions advised pelvic floor exercises don't do when person had surgery and must wait at least 6 to 8 weeks before do kegel's exercises because the body needs time to heal, had a urinary catheter insertion because may cause bleeding or bladder spasms, during medical pelvic exams and when person feel pain during sex. Canadian Guideline for Physical Activity (2019) recommended that there are absolute contraindications to exercise such as; ruptured membranes, premature labour, unexplained persistent vaginal bleeding, placenta previa after 28 weeks' gestation, preeclampsia, incompetent cervix, intrauterine growth restriction, high order multiple pregnancy (e.g., triplets), uncontrolled type one diabetes, uncontrolled hypertension, uncontrolled thyroid disease and other serious cardiovascular, respiratory, or systemic disorder. in addition there are relative contraindications to exercise such as; recurrent pregnancy loss, gestational hypertension, a history of spontaneous preterm birth, mild/moderate cardiovascular or respiratory disease, symptomatic anemia, 
malnutrition, eating disorder, twin pregnancy after the 28th week and other significant medical conditions (Canadian Guideline for Physical Activity (11).

\section{Technique of pelvic floor muscles exercise}

There are some instruction should be perform before doing kegel's exercise; the woman should sure bladder completely empty before doing Kegel's exercises. Very important to be focus on tightening the pelvic floor muscles only when performing kegels exercise and don't move or squeeze abdominal muscles, the legs and buttock during kegels; take breathing normally during exercising; don't try to do pelvic floor muscles exercise while sneezing or coughing to prevent disturbing situations (4).

\section{The following the technique for kegel's exercise:}

The woman sits down and tendency lightly forward with upright back; Start with recognizing the right pelvic floor muscles. Squeeze the muscles as if the woman is attempting to stop the urine and passing of gas with each other; in case the woman is disabled to insulate the muscles at first, introduce a clean finger into the vagina, if the woman feel pressure on the finger, she has got correct muscles. Finally, hold the contraction for eight to twelve counts and then relax for the same counts. Repeat two to three sets sundry times daily (12).

\section{Exercise of pelvic floor muscles}

The first exercise: The women squeeze the pelvic muscles for three seconds; relax the muscles for three seconds; then squeeze again. If it is complex to hold for three seconds, attempt two seconds and setting up as the muscles obtain powerful. Purpose for a chain of ten squeezes and releases, three times daily $(4,12)$.

The second exercise: The flutter exercise is such as the first excluding that in state holding the squeeze for three seconds, the aim is to squeeze the muscle, release it, squeeze still and release as speedily as potential. Again, complete a chain of ten squeezes and releases at three unlike times at a day $(4,12)$.

The third exercise: The total length of the vagina (pelvic muscles and uterine) including in this exercise. The woman visualized the vagina is an elevator shaft and the elevator is at the orifice to the vagina. Contract the muscles as visualizing her slowly drawing the elevator upward, forever the vaginal canal, starting at the beginning and ending at the uterus. After the three to four seconds it occupies to repair the entire length of the vagina, slowly relax the muscles as if woman was lowering the elevator to the ground floor, and then start again at the vaginal orifice. Make three chains of ten contractions every day. This exercise is perfect for strengthening the uterine muscles, as well as the pelvic muscles $(12,13)$.

\section{Effects of Kegel's exercise training during pregnancy on labor outcome and postpartum}

There is broad evidence suggesting that in a healthy pregnant woman, exercise has several benefits for both mental and physical. As long as the pregnant woman hasn't side effects through the pregnancy period, nearly any kind of exercise can be done of course this does demand a common feel approach and avoidance of activities that can lead to breakdowns. Since pregnant women are routinely followed up by a primary care provider or prenatal nurse, these professionals are in the model position to instruct the pregnant woman on the benefits of exercise (14).

The antenatal Kegel's exercise was effective when pregnant women done it at 34 weeks of gestational age can be helped in promotion of uterine contraction and improved the perineum to be an intact after vaginal delivery (15).

In addition, the researchers founded that kegel's exercise is consolidating delivery through vaginal between pregnant women, inexpensive drawing for lowering incidence 
of caesarean section, safety and nonpharmacological methods. Also, the antenatal pelvic floor muscles training exercise could be important benefit to pregnant women in reduce rate of prolonged second stage of labor and incidence of episiotomy $(1,16)$.

The researchers when done a quasirandomized controlled trial to assess influence of a pelvic floor training program to prevent perineal trauma, the researchers founded that pelvic floor muscles exercises in primiparous women increasing an intact perineum, decreases rates of episiotomy, reduces severe perineal trauma and reduce perineal pain in postpartum period. Also, they recommended to all women must be done kegal's exercise at 32 weeks of pregnancy to prevent perineal trauma (17).

The kegel's exercise training during third trimester helping in strengthen pelvic floor muscles during pregnancy can help the muscles control well during labor and delivery. Also helping in reduce duration of labor, reducing episiotomy rate, decrease in the incidence of perineal tear or laceration and decrease in the incidence of cesarean section (18).

The researcher when done a controlled randomized study to assessment of fetal wellbeing in pregnant women subjected to pelvic floor muscle training. They conducted that, pelvic floor muscle training in low-risk primigravida with singleton pregnancy was associated with a significant decline in pulsatility index (PI) of the uterine artery after exercise, while no significant changes in middle cerebral and umbilical flow were found, and may be safely recommended for the prevention and treatment of urinary incontinence (19).

The researchers when done a systematic review with meta-analysis to assess antenatal kegel's exercise training on labor and delivery outcomes, the researchers founded that pelvic floor muscles exercises, it has an important benefit to women during pregnancy period in reducing the first and second stage of labor in the primigravida. That the 1 st and 2 nd stage of labor was shortened by about twenty eight minutes and ten minutes on average may signalize an important clinical significance (20).

In addition, the researchers when done a systematic review to determine the effects of pelvic floor interventions during pregnancy on childbirth related and pelvic floor parameters the researchers founded that, the several clinical trials showed evidence that kegel's exercise shortens the second stage of labor and decrease the risk of leakage of urine during pregnancy period. Therefore, kegel's exercise should be taught routinely during antenatal care and also, should be practiced during pregnancy period at home (21).

Also, there are some researches recommended that, the beginning of doing pelvic floor muscles exercise training at 28- 32 weeks of pregnancy because eight to twelve weeks are the maximum time of muscles training to prevent hypertrophy to the pelvic floor muscle. Also in this time the perineal muscle increase in flexibility and increase in stretching at birth without perineal trauma (22).

\section{References}

1) Abd-El Hamid, A.A., Azzam, H.F., Ismail, G.M., \& Gaafar, H.M,. (2012): Effect of a structured antenatal kegel exercises protocolon labor progress among women attending antenatal clinics. Egyptian Nursing Journal; 3, 2090-2106.

2) Bo, K., Hilde, G., Stær-Jensen, J., Siafarikas, F., Tennfjord, M.K., \& Engh, M.E., (2015): Postpartum pelvic floor muscle training and pelvic organ prolapse: a randomized trial of primiparous women. Am J Obstet Gynecol. 212(1): 38.e1-38.e7.

3) Yeniel AO, Petri E (2014). Pregnancy, childbirth, and sexual function: perceptions and facts. IntUrogynecol J. 2014; 25(1):5-14.

4) American Academy of Family Physicians (2019): Kegel exercises for 
your pelvic muscles. Available at; https://www.clinicalkey.com. (Accessed at January 04, 2019)

5) Kegel, A.H,. (1948): Progressive Resistance Exercise to the Functional Restoration of the Perineal Muscles. Ame. J. Obst. Gynec. 56(2): 238-248.

6) Bekhatroh, A., (2017): Effect of Pelvic Floor Muscles Exercises Program on Women's Sexual Selfefficacy after Delivery. Madridge J Nurs. 2(1): 48-55.

7) Due, U., Klarskov, .N, Gras, S., \& Lose, G,. (2019): Pelvic floor muscle training with and without supplementary KAATSU for women with stress urinary incontinence - a randomized controlled pilot study Neurourol Urodyn. 38(1):379-386.

8) ElDeeb, A.M., Abd-Ghafar, K.S., Ayad, W.A., \& Sabbour, A.A., (2019): Effect of segmental stabilizing exercises augmented by pelvic floor muscles training on women with postpartum pelvic girdle pain: A randomized controlled trial. Journal of Back and Musculoskeletal Rehabilitation; $1-8$. DOI:10.3233/BMR-181258.

(Accessed at January 4, 2019)

9) Nazarpour, S., Simbar, M., Tehrani, F.R., \& Majd, H.A,. (2017): Effects of Sex Education and Kegel Exercises on the Sexual Function of Postmenopausal Women: A Randomized Clinical Trial. J Sex Med.14:959-967.

10) Lucio, A.C., Lourenço, CH.B., Damasceno, B.P., Lopes, M.H.B., \& D'ancona C.A.L., (2018). The effect of pelvic floor muscle contraction on detrusor overactivity pressure in neurogenic and non-neurogenic women during urodynamic study. American Journal of Physical Medicine \& Rehabilitation; DOI: 10.1097/PHM.00000000000010 65 (Accessed October 19, 2018).

11) Canadian Guideline., (2019): Canadian guideline for physical activity through pregnancy. Retrieved from https://csepguidelines.ca/guidelinesfor- pregnancy/ (Accessed June 19, 2019).

12) Tommy's, (2017): Pelvic floor exercise: Available from https//WWW.tommys.org/regnancy. Information/im-pregnant/exercise pregancy/pelvic-exercise ( Accessed April 17, 2018).

13) National Health Service Choices, (2017): Exercise in pregnancy. Available from;http://WWW.nk/conditions/preg nancy.and-baby/pages/pregnancyexercise.aspx(Accessed31 October 2017).

14) Ceprnja D., \& Gupta, A., (2018). Does muscle energy technique have an immediate benefit for women with pregnancy-related pelvic girdle pain? Physiother Res Int. 12; e1746.

15) Alves, P.G.J.M., Cavalli, R.C., Bertacini, D.M.M., \& Driusso, P,. (2016): Effects of antenatal pelvic floor preparation techniques on the perineal integrity: a randomized controlled trial protocol. International Journal of Clinical Trials; 3(4):203209.

16) El-Shamy, F., \& Abd El Fatah, E, (2018): Effect of Antenatal Pelvic Floor Muscle Exercise on Mode of Delivery: A Randomized Controlled Trial. Integr Med Int;4:187-197.

17) Larios, F. L., Gutierrez, I. C., Mejía, R. C., \& Serrano, S. C,. (2017): Influence of a pelvic floor training programme to prevent perineal trauma: A quasi-randomised controlled trial. Midwifery journal; 50: 72-77.

18) Goda, A .A., Fawaz, M. A., \& Ghonem, G,. (2015): Effect of pelvic floor muscle exercise training protocol for pregnant woman during 3rd trimester on labor duration. An International Peer-reviewed Journal; 15: 10-19.

19) Okido, M.M., Valeri, F.L., Martins, W.P., Ferreira, C.H., Duarte, G., \& Cavalli, R.C,. (2015): Assessment of fetal wellbeing in pregnant women 
subjected to pelvic floor muscle training: A controlled randomised study. Int Urogynecol J; 26: 14751481.

20) Du, Y., Li, X.u., L., Ding, L., Wang, Y., \& Wang, Z,. (2015): The effect of antenatal pelvic floor muscle training on labor and delivery outcomes: a systematic review with meta-analysis. Int Urogynecol J; 26:1415-1427.

21) Schreiner, L., Crivelatti, M., Oliveira, J.M., Nygaard, C.C., \& Santos, T,G,. (2018): A Systematic review of pelvic floor interventions during pregnancy. Int J Gynecol Obstet; 143: 10-18.

22) Dumoulin, C., Glazener, C., \& Jenkinson, D,. (2011): Determining the optimal pelvic floor muscle training regimen for women with stress urinary incontinence. Neurourol Urodyn; 30(5):746-53. 
